\title{
Trois nouvelles Onchocerques chez des Céphalophes du Gabon
}

\author{
par O. BAIN, A.-G. CHABAUD et I. LANDAU * \\ Laboratoire de Zoologie (Vers), associé au C.N.R.S., Muséum national d'Histoire naturelle, \\ 43, rue Cuvier, F 75231 Paris Cedex 05.
}

\section{Résumé.}

Les Céphalophes de la région de Makokou au Gabon hébergent de nombreuses espèces d'Onchocerques mais les adultes ne peuvent être découverts que par une recherche systématique.

Trois nouvelles espèces sont décrites : $O$. sonini n.sp. dans les tendons des pattes et O. lerouxi n.sp. dans les aponévroses musculaires du tronc chez Cephalophus nigrifrons, $O$. beaveri n.sp. dans les ligaments de l'articulation atlas-occiput chez $C$. monticola. Bien que d'aspect extérieur très différent, ces trois espèces appartiennent à un seul groupe dont le niveau d'évolution morphologique se situe entre les Onchocerques d'Equidés, plus primitives, et les Onchocerques des autres animaux, plus évoluées.

\section{Summary.}

Three new species of Onchocerca from duikers in Gabon.

The duikers from Makokou (Gabon) are parasitized by many species of Onchocerca but the adults are very often occult and can only be discovered by a systematical search.

Three new species are described : $O$. sonini n.sp. in the feet-tendons, and $O$. lerouxi $\mathrm{n} . \mathrm{sp}$. in the muscular aponevrosis of the trunk of $C$. nigrifrons; O. beaveri n.sp. in the ligaments of the atlas-occipital articulation of C. monticola.

Although their external appearance differs, these three species belong to a single group, which, from an evolutionary point of view, is intermediate between the more primitive Onchocerca of Equiids and those more evolved from other animals.

* Ce travail a pu être fait grâce à une subvention de l'Organisation mondiale de la santé. Accepté le 28 décembre 1977. 


\section{Introduction}

Le potentiel annuel de transmission (c'est-à-dire le nombre de stades infestants inoculés à un individu par an) prend une importance prédominante dans les recherches modernes sur l'Onchocercose humaine. Les troisièmes stades infestants ayant une morphologie très homogène, quelle que soit l'espèce en cause, et Simulium darnnosum sensu lato se nourrissant fréquemment sur des animaux aussi bien que sur l'homme, il devient essentiel de connaître les onchocercoses des animaux sauvages, particulièrement en zone d'endémie humaine.

Le Roux, et d'autres auteurs, ont montré depuis longtemps l'existence de ces Onchocerques; les références de ces travaux sont données par Sonin (1975). Les excellents résultats obtenus par digestion pepsique sur le matériel fixé à l'alcool et les progrès faits dans l'étude systématique de ces Filaires permettent de reprendre le problème de façon plus précise.

Un sondage effectué sur quelques Céphalophes de forêt dans la région de Makokou au Gabon nous a surpris par la fréquence des résultats positifs.

La localisation très élective des microfilaires et les lésions extrêmement discrètes déterminées par les adultes font que ces Onchocerques échappent facilement à l'examen. En outre, certains des animaux dont nous avons disposé étant fortement putréfiés, des Onchocerques ont inévitablement échappé à nos recherches. Cependant, sur sept spécimens examinés, les résultats sont les suivants :

- Cephalophus monticola Thunb: deux spécimens; dans les deux cas, les biopsies cutanées des oreilles révèlent des centaines de microfilaires; en revanche, dans le reste du corps, nous n'obtenons que des prélèvements faiblement positifs : cou, six microfilaires ; doigt postérieur, une microfilaire. Dans les deux cas, des Onchocerques ayant des microfilaires apparemment identiques ont été trouvées dans le ligament articulaire reliant l'atlas à l'occiput ( $O$. beaveri n. sp.).

- Cephalophus dorsalis Gray : sur deux spécimens, l'un a, dans les snips effectués au niveau des cuisses, un petit nombre de microfilaires de type Onchocerque, remarquablement longues et fines. Aucun adulte correspondant n’a été décelé.

- Cephalophus callipygus Peters: 1 spécimen n’a montré que de rares microfilaires de type Onchocerque dans les oreilles; l'autopsie détaillée de la tête du Céphalophe a été négative.

- Cephalophus nigrifrons Gray: deux spécimens ont été autopsiés; l'un a montré quelques microfilaires de type Onchocerque dans les oreilles et le cou mais l'autopsie détaillée de la tête a été négative. Dans l'autre spécimen au contraire tous les snips examinés (museau, oreille, cou, dos, flanc, ventre cuisse, pattes) ont été négatifs, alors que l'autopsie a révélé deux nouvelles espèces : $O$. sonini $\mathrm{n}$. sp., dans les tendons des pattes et $O$. lerouxi n. sp. dans les aponévroses musculaires profondes. 


\section{Onchocerca sonini n. sp.}

Cette Onchocerque, trouvée chez Cephalophus nigrifrons, est située en haut de l'os canon, sur la face antérieure (pattes antérieures), sur la face postérieure (pattes postérieures), entre le tendon superficiel et le derme; les quatre membres sont parasités. Elle forme des kystes allongés, plus ou moins transparents. Les Filaires sont dans un tunnel un peu scléreux, très contourné, qui forme à chacune un étui particulier. Il existe parfois des kystes plus petits à proximité des kystes principaux ; l'un deux, peu sclérifié, renferme une femelle juvénile. Un mâle libre est récolté à la dissection; un autre après digestion pepsique.

\section{Matériel :}

Une femelle entière holotype, un mâle allotype ainsi qu'un autre mâle et une extrémité antérieure de mâle, trois femelles entières, huit régions antérieures de femelles ainsi que de nombreux fragments du corps (lot 292 PB, oncho 1). Malgré l'abondance du matériel, les microfilaires sont rares comme si la plupart des femelles n’avaient pas été fécondées.

\section{Femelle (fig. 1 et 2).}

La région antérieure est mince $(105 \mu \mathrm{m})$ à la fin de l'œsophage musculaire et faiblement ondulée. Le reste du corps est plus épais (jusqu'à $250 \mu$ ) et forme des ondulations lâches et irrégulières, souvent plus serrées vers la queue.

Dans la partie moyenne du corps, la musculature est épaisse et la cuticule également ; celle-ci atteint 30 à $50 \mu \mathrm{m}$ d'épaisseur et est formée de trois couches (fig. $2, \mathrm{~A}$ ). La couche externe est épaisse et a une structure fibreuse, orientée longitudinalcment ; elle forme des côtes droites, espacées de 38 à $58 \mu \mathrm{m}$, interrompues dans le plan latéral. La couche moyenne, également épaisse, apparaît fibreuse en coupe transversale ; en vue superficielle, elle est striée, au nombre de quatre stries par intercôte ; les stries sont interrompues dans le plan latéral (fig. 2, B) ; parfois cette couche cuticulaire est ornée, entre les stries, de massifs grumeleux (fig. 2, C). La couche externe et la couche moyenne sont plus épaisses dans les champs latéraux et se subdivisent chacune à ce niveau en deux couches. La couche interne est la plus mince ; elle est très brillante et de structure homogène.

Aux extrémités du corps, la cuticule n'est formée que par deux couches. A $1 \mathrm{~cm}$ de l'apex (fig. 1, H), les côtes externes sont déjà bien visibles mais elles sont peu espacées et il n'y a pas de stries. Celles-ci apparaissent à $2 \mathrm{~cm}$ de l'apex (fig. $1, I$ ).

Sur la tête, les papilles ont une disposition figurée en $1, B, C$, et $D$. Un renflement circulaire, plus accentué ventralement, marque l'emplacement de l'anneau nerveux et du pore excréteur. La vulve, située vers le début de l'œsophage glandulaire, est saillante. 


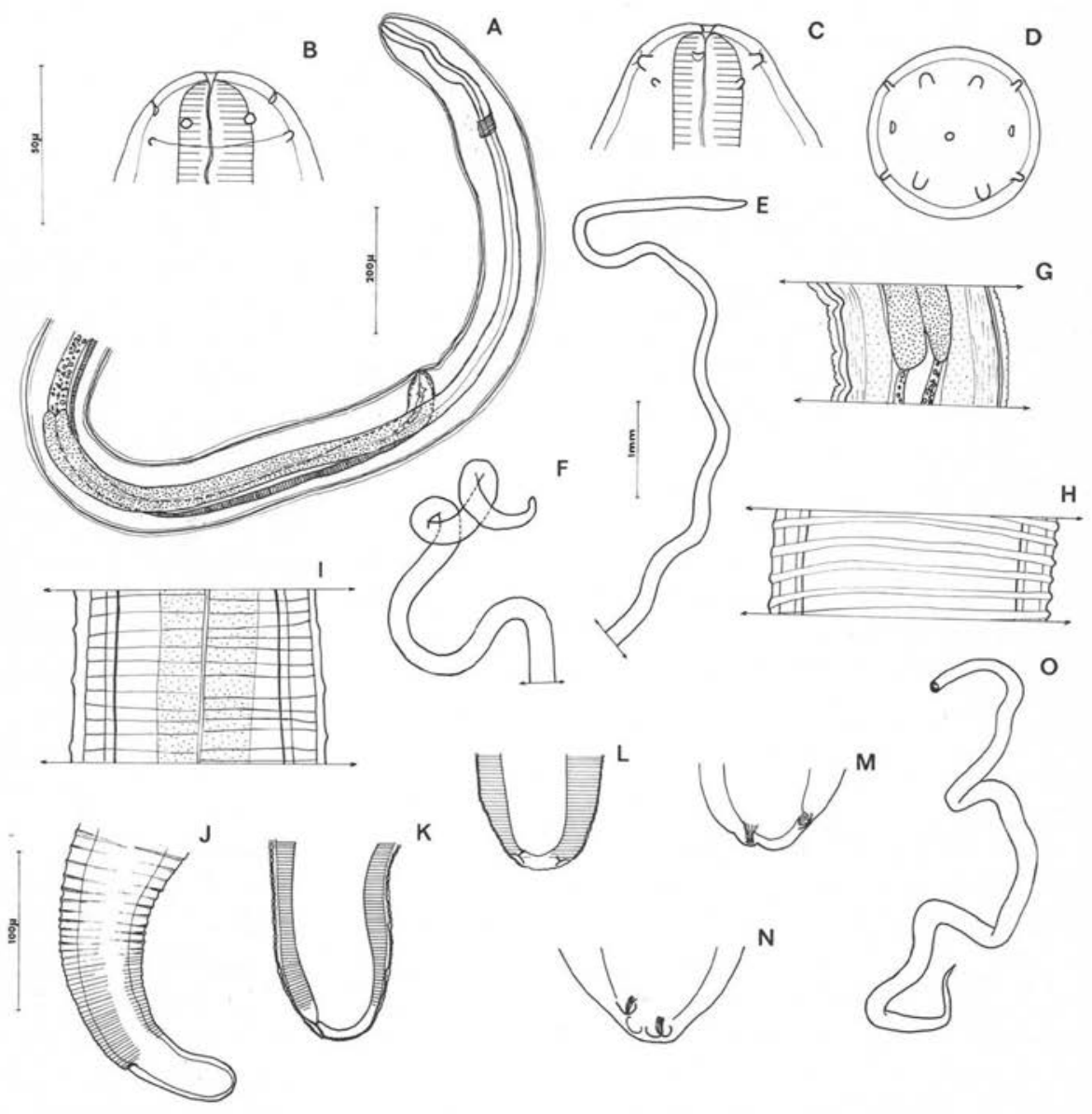

FIG. 1. - Onchocerca sonini n. sp.; femelle. A : region antérieure, vue latérale ; B, C, D : tête en vue médiane, latérale et apicale; $\mathrm{E}$ et $\mathrm{F}$ : silhouettes des régions antérieure et postérieure; $\mathrm{G}$ : jonction œsophage-intestin; $\mathrm{H}$ : cuticule à $1 \mathrm{~cm}$ de la tête ; $\mathrm{I}$ : idem, à $2,5 \mathrm{~cm}$ de la tête; $\mathrm{J}$ : région caudale, vue latérale; $\mathrm{K}$ et $\mathrm{L}$ : extrémité caudale en vues latérale et ventrale; $\mathrm{M}$ et $\mathrm{N}$ : idem, autres femelles; $\mathrm{O}$ : microfilaire utérine.

(A et J : éch. $200 \mu ; \mathrm{B}, \mathrm{C}, \mathrm{D}, \mathrm{M}, \mathrm{N}, \mathrm{O}:$ ech. $50 \mu$;

$\mathrm{E}$ et $\mathrm{F}$ : éch. $1 \mathrm{~mm} ; \mathrm{G}, \mathrm{H}, \mathrm{I}, \mathrm{K}, \mathrm{L}$ : éch. $100 \mu)$.

La région caudale est brusquement amincie; la queue est souvent incurvée vers la face dorsale; son extrémité, assez variable, est arrondie ou conique-obtuse et porte les deux phasmides, souvent dissymétriques.

Dimensions. - Femelle holotype : corps long de $145 \mathrm{~mm}$ et large de $210 \mu \mathrm{m}$; anneau nerveux à $330 \mu \mathrm{m}$ de l'apex; œsophage long de $1310 \mu \mathrm{m}$; vulve à $830 \mu \mathrm{m}$; queue longue de $125 \mu \mathrm{m}$ et large à l'anus de $80 \mu \mathrm{m}$. 


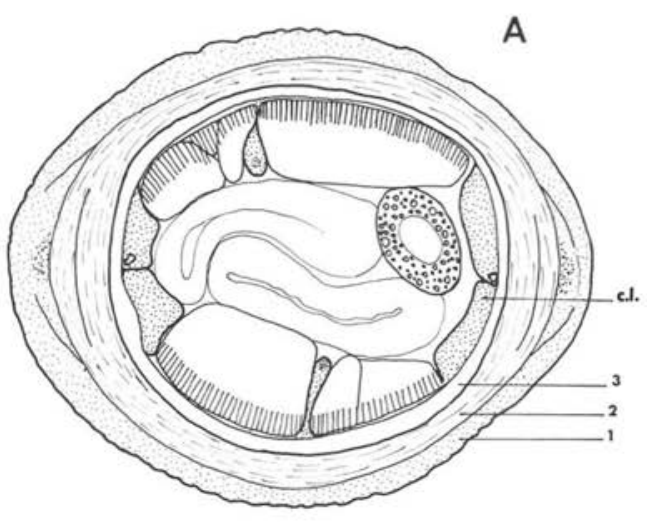

B

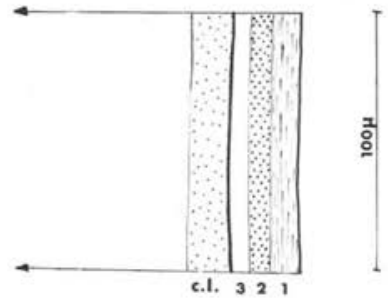

D
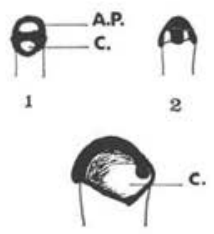

4

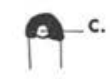

3

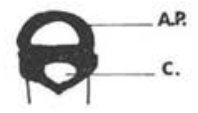

5

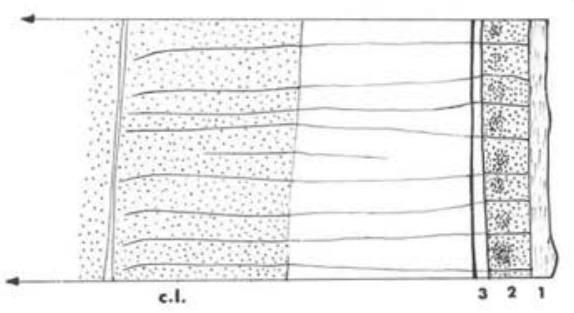

FIG. 2. -- Onchocerca sonini n. sp.; femelle. A : coupe transversale du corps; B, vers le même niveau, cuticule du corps en vue longitudinale médiane ; $\mathrm{C}$ : idem, en vue longitudinale latérale (autre région du corps) (C. 1 champ latéral 1, 2 et 3 : couches externe, moyenne et interne de la cuticule); D : tête de la microfilaire, 1 et 5 , vue apicale, 2, vue perpendiculaire au plan du crochet; 3 , crochet vu de face, 4 , crochet en vue oblique. (A, B, C, éch. $100 \mu ; \mathrm{D}$, à main levée).

Autres femelles : anneau nerveux, pore excréteur et vulve à 240,300 et $760 \mu \mathrm{m}$ de l'apex; œsophage long de $1480 \mu \mathrm{m}$ avec portion glandulaire de $780 \mu \mathrm{m}$; cuticule épaisse de 30 à $50 \mu \mathrm{m}$ (épaisseur maximale dans le plan latéral); intercôtes de $38-58 \mu \mathrm{m}$. Queues longues de $160 \mu \mathrm{m}, 180 \mu \mathrm{m}$ et $150 \mu \mathrm{m}$ et larges de $80 \mu$ à l'anus.

\section{Microfillaires (fig. $1, O$ et fig. 2, D).}

La microfilaire a un corps large dans la partie moyenne et une région antérieure très effilée, avec une tête petite et ronde dont l'aspect est tout à fait remarquable : elle est recouverte par un capuchon cuticulaire épais et très réfringent, si bien que les utérus mûrs apparaissent piquetés de grains brillants. Suivant l'orientation de la tête, ce capuchon se présente sous différents aspects (fig. 2, D). Pour obtenir une interprétation cohérente de ces images, les têtes des microfilaires ont été tournées dans différentes positions sous le microscope. Il est apparu que l'aspect très original 
de ce capuchon résulte en fait de l'hypertrophie de structures cuticulaires céphaliques simples tout à fait comparables à celles qui ont été mises en évidence chez O. volvulus par Laurence et Simpson (1968). Dans les deux cas, la tête porte un anneau péribuccal et un crochet mais, alors que chez $O$. volvulus ces deux structures ne sont révélées clairement que par une coloration spéciale, elles sont puissantes et directement visibles chez notre microfilaire: les figures $D 1$ et 5 représentent la tête en vue apicale avec en haut l'anneau péribuccal et en bas, le crochet très développé dont la partie centrale, transparente, offre l'apparence d'un orifice ; les figures 2, 3 et 4 représentent des vues de la tête sous trois angles différents : dans le plan perpendiculaire au crochet (2), le crochet, transparent au centre, vu de face (3), le crochet, en vue oblique, montrant son attache sur la barre cuticulaire en forme d'arc (4).

Dimensions. - Une seule microfilaire a pu être extraite entière des utérus : corps long de $233 \mu \mathrm{m}$ et large de $7 \mu \mathrm{m}$ au milieu du corps ; queue courte et conique.

\section{MÂLE (fig. 3).}

Le renflement cervical est également présent. La cuticule du corps, formée de deux couches, est striée (fig. 3, B). La queue est renforcée à l'apex par une large pointe conique. La disposition des papilles est figurée en $3, D$ et $E$; les phasmides sont situées près de l'avant dernière paire de papilles; la dernière paire est transformée en pointe cuticularisée. Le spicule droit a un talon peu marqué; le spicule gauche se termine par une pointe courte.

Dimensions. - Mâle allotype : corps long de $19,8 \mathrm{~mm}$ et large de $120 \mu \mathrm{m}$; anneau nerveux à $260 \mu \mathrm{m}$ de l'apex ; œsophage long de $1025 \mu \mathrm{m}$; queue, spicule gauche et spicule droit longs de $110 \mu \mathrm{m}, 180 \mu \mathrm{m}$ et $98 \mu \mathrm{m}$.

Deuxième mâle : corps long de $15,9 \mathrm{~mm}$ et large de $87 \mu \mathrm{m}$; anneau nerveux et pore excréteur à $230 \mu \mathrm{m}$ et $265 \mu \mathrm{m}$, œsophage long de $1030 \mu \mathrm{m}$ avec portion glandulaire de $580 \mu \mathrm{m}$; queue, spicule gauche et spicule droit longs de $98 \mu \mathrm{m}, 213 \mu \mathrm{m}$ (manche de $100 \mu \mathrm{m})$ et $88 \mu \mathrm{m}$.

\section{Discussion.}

Cette filaire est immédiatement reconnaissable par les structures cuticulaires céphaliques hypertrophiées de la microfilaire (anneau péribuccal et crochet) qui simulent un capuchon recouvrant la tête ; nous n'en connaissons pas d'équivalent chez les autres onchocerques.

Par l'œsophage à portion glandulaire bien différenciée, nos spécimens peuvent être rapprochés de six espèces d'Onchocerques : trois sont parasites d'Equidés, O. reticulata Diesing 1841, O. cervicalis Railliet et Henry 1910 et O. raillieti Bain, Muller et coll., 1976; une est parasite de Bovins, O. armillata Railliet et Henry, 1909 et deux de Cervidés, O. flexuosa (Wedl, 1856) et O. tubingensis Bain et Schulz-Key, 1974. 


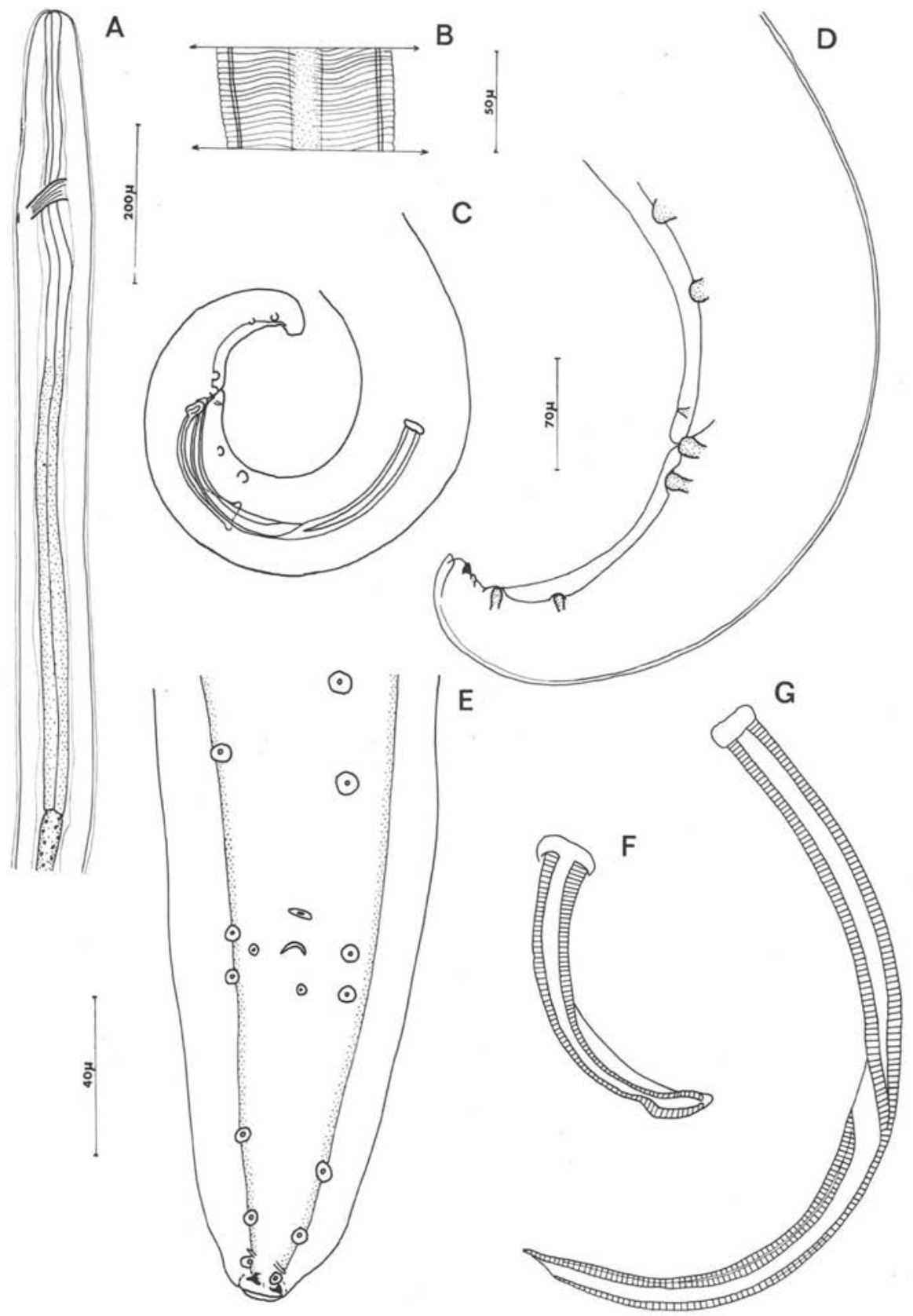

FIG. 3. - Onchocerca sonini n. sp. Mâle. A : région antérieure, vue latérale; B : cuticule au milieu du corps, vue latérale; C : région caudale, vue latérale ; D : queue, vue latérale ; $\mathrm{E}$ : idem, vue ventrale ; $\mathrm{F}$ et $\mathrm{G}$ : spicules droit et gauche, vue latérale. (A. éch. $200 \mu, \mathrm{B}$, éch. $50 \mu, \mathrm{C}$, éch. $70 \mu, \mathrm{D}, \mathrm{E}, \mathrm{F}, \mathrm{G}$ : éch. 40 ॥). 
Toutefois ces six espèces diffèrent de notre matériel par l'absence de renflemeni cervical et par plusieurs autres caractères propres à chaque espèce :

- O. reticulata : corps plus large, œsophage trois à quatre fois plus long, côtes et intercôtes de même largeur, papilles labiales externes sur les axes qui vont de la bouche aux papilles céphaliques, groupe terminal des papilles caudales réduit à une seule paire.

- O. cervicalis : œsophage également plus long, cuticule de la femelle à deux couches, trois stries par intercôte, dernière paire de papilles caudales non transformée en pointe cuticulaire.

- O. raillieti : côtes absentes sur la partie moyenne du corps, papilles caudales au complet (21).

- O. armillata : côtes ondulées, queue très longue chez la femelle, papilles caudales disposées en deux files longitudinales.

- O. flexuosa : très grandes dimensions dans les deux sexes, papilles caudales toutes post-cloacales.

- O. tubingensis: œsophage très court, groupe terminal de papilles réduit à deux paires non aciculées.

L'Onchocerca sp. récoltée par Le Roux en 1947 chez un Céphalophe en Zambie (Sylvicapra grimmia) est très incomplètement connue, mais, par la cuticule très mince et les côtes très étroites (cf. Bain et Muller, 1978), elle ne peut être assimilée à ce matériel.

Nos spécimens s'opposent donc aux Onchocerques connues et forment une nouvelle espèce que nous dédions à notre collègue $\mathrm{M}$.-D. Sonin, en la nommant O. sonini n. sp.

Elle se caractérise par l'œsophage glandulaire développé, les côtes droites séparées par quatre stries, la cuticule épaisse divisée en trois couches, la dernière paire de papilles caudales aciculée, la microfilaire au corps épais et à tête fine recouverte d'un capuchon cuticulaire.

\section{Onchocerca lerouxi n. sp.}

Cette Onchocerque est située entre les plans musculaires du thorax, de l'abdomen et des cuisses de Cephalophus nigrifrons. La lésion est à l'intérieur d'une lame élastique d'aponévrose ; elle se présente sous l'aspect de cordons sinueux, souvent terminés par une petite masse arrondie, de couleur blanc jaunâtre et qui se confond aisément avec des filets graisseux.

L'Onchocerque est à l'intérieur de ces cordons; son corps adhère aux parois du tunnel. 


\section{Matériel :}

Une femelle entière avec microfilaires (holotype), un mâle (allotype), sept extrémités antérieures de femelle, ainsi que plusieurs fragments de corps femelles dont certains avec microfilaires (lot 292 PB, Oncho 2).

\section{Femelle (fig. 4).}

La région antérieure est mince (120 $\mu \mathrm{m}$ à la fin de l'œsophage musculaire) ; en arrière, il existe une longue portion qui est à peu près rectiligne sur $5 \mathrm{~cm}$ et dont le diamètre augmente lentement jusqu'à $150 \mu \mathrm{m}$. Le reste du corps est épais (325 $\mu \mathrm{m})$ et forme des sinuosités irrégulières, situées dans un seul plan, celui de l'aponévrose. En plus de ces sinuosités, le corps présente une torsion en spirale par rapport à l'axe longitudinal de telle sorte que les lignes latérales sont, à certains niveaux tout au moins, obliques par rapport aux côtes et aux stries.

Dans la région moyenne du corps, la cuticule est épaisse de 15 à $22 \mu \mathrm{m}$, suivant son degré d'étirement. Elle est formée de deux couches, sensiblement de même épaisseur; la couche externe porte des côtes rectilignes espacées de 30 à $55 \mu \mathrm{m}$, peu saillantes et difficiles à voir de face ; la couche interne est nettement striée, à raison de quatre stries en général par intercôte, rarement cinq. La musculature est mince : les champs latéraux sont traversés par deux volumineux canaux excréteurs (fig $4 \mathrm{~K}$ et $5 \mathrm{~J}$ ).

Dans la région antérieure du corps, la cuticule n'est formée que par une couche, régulièrement ondulée au niveau de l'œsophage glandulaire ; à $1 \mathrm{~cm}$ de la tête, la couche interne apparaît, elle est mince; en même temps les côtes se forment sur la couche externe (fig. 4).

Dans la région postérieure, la cuticule est formée d'une seule couche, mais elle est épaisse et ornée de côtes de plus en plus serrées et de plus en plus fines vers l'extrémité caudale (fig. 4).

Sur la tête, la disposition des papilles est figurée en $4, B, C$ et $D$; le pore excréteur est situé sous une légère protubérance; la vulve est située vers la fin de l'œsophage musculaire.

La région postérieure est cônique, la queue elle même est presque cylindrique, son extrémité est ornée par trois petites languettes arrondies : une terminale et deux latérales ventrales où arrivent les phasmides (fig. $4, L, M$ et $N$ ).

Dimensions. - La femelle holotype est longue de $255 \mathrm{~mm}$ et large au maximum de $325 \mu$; anneau nerveux, pore excréteur et vulve à 220,250 et $570 \mu \mathrm{m}$ de la tête; œsophages musculaire et glandulaire longs de 620 et $1030 \mu \mathrm{m}$; queue longue de $230 \mu \mathrm{m}$ et large de $120 \mu \mathrm{m}$ à l'anus.

Chez trois femelles les régions antérieures ont les dimensions suivantes : anneau nerveux à 215,240 et $200 \mu \mathrm{m}$ de la tête; pore excréteur à 245,270 et $235 \mu \mathrm{m}$ de la tête ; vulve à 480,650 et $520 \mu \mathrm{m}$ de la tête ; œsophage musculaire long de 700,620 et $550 \mu \mathrm{m}$; œsophage glandulaire long de 1200,1360 et $1240 \mu \mathrm{m}$. 


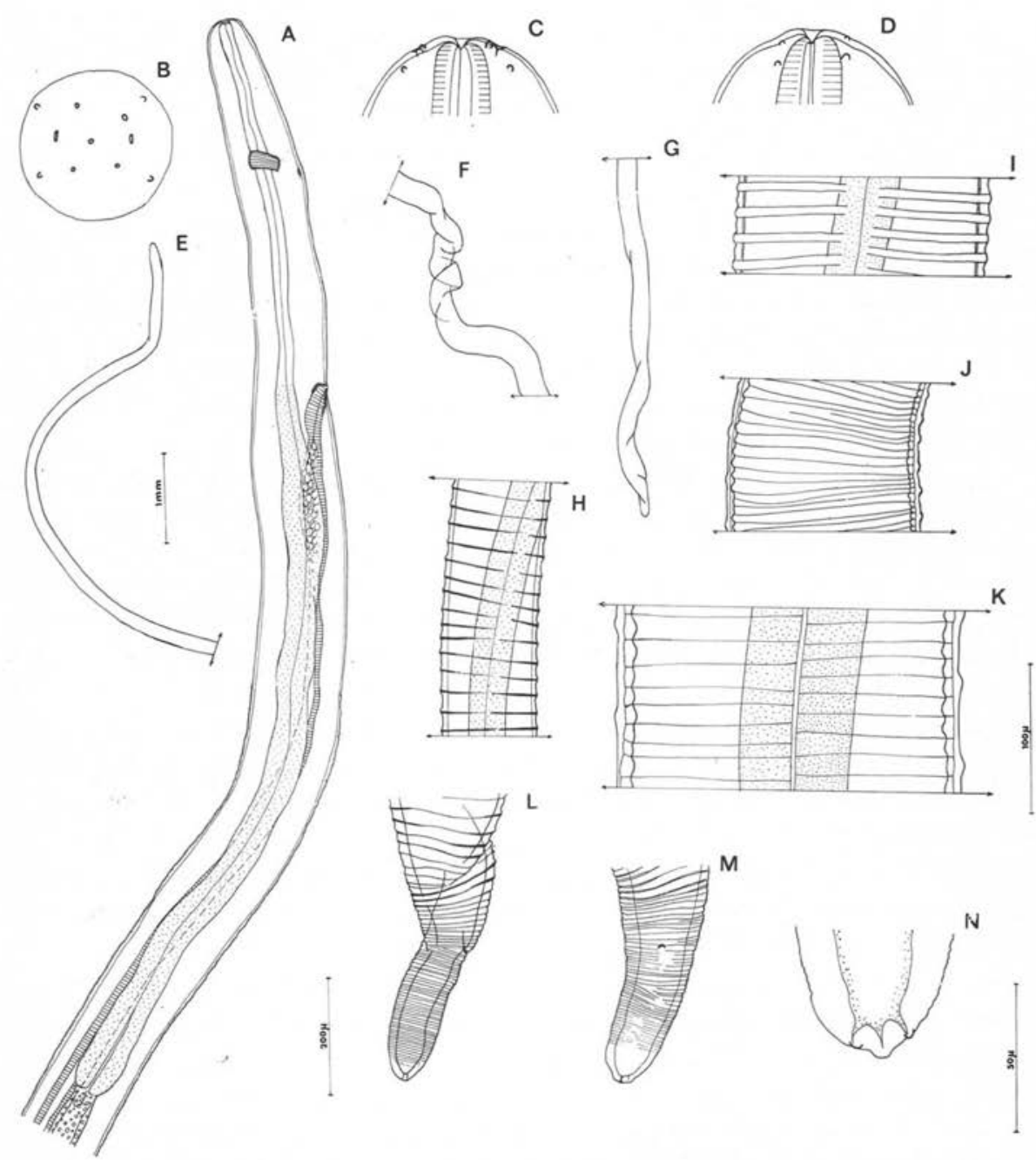

FIG. 4. - Onchocerca lerouxi n.sp.; femelle. A : région antérieure, vue latérale; B, C et D : tête, vues apicale, médiane et latérale ; E, F et $\mathrm{G}$ : silhouettes des régions antérieure, moyenne et postérieure; $\mathrm{H}$, torsion du corps sur son axe et disposition des côtes ; I : cuticule à $1 \mathrm{~cm}$ de la tête; $\mathrm{J}$ et $\mathrm{K}$ : cuticule dans la région moyenne du corps (les côtes, très transparentes, ne sont pas représentées de face); $\mathrm{L}$ : région caudale, vue latérale; $\mathbf{M}$, idem, vue ventrale (l'extrémité caudale est vue de profil); $\mathbf{N}$ : extrémité caudale, vue ventrale.

(A, H, J, L et M : éch. $200 \mu ; \mathrm{B}, \mathrm{C}, \mathrm{D}$ et $\mathrm{N}$, éch. $50 \mu$; $\mathrm{E}, \mathrm{F}$ et $\mathrm{G}$ : éch. $1 \mathrm{~mm}$, I et $\mathrm{K}$ : éch. $100 \mu)$. 
Microfilaire (fig. $5 \mathrm{G}$ ).

Les microfilaires utérines sont grêles (3 à $3,5 \mu \mathrm{m}$ ), elles sont longues de 255 à $290 \mu \mathrm{m}(290,268,295,278,275,256,270,290,270$ et $255 \mu \mathrm{m})$; la région antérieure est amincie ; la tête est arrondie et plus étroite que le corps, ou légèrement renflée, suivant l'orientation; le crochet est à peine perceptible ; la pointe caudale est courte, souvent recourbée, et porte un mucron terminal très étroit.

MâLE (fig. 5).

Le mâle paratype est grêle et mesure $21,7 \mathrm{~mm}$ de long sur $145 \mu \mathrm{m}$ de large. Anneau nerveux et pore excréteur à 255 et $270 \mu \mathrm{m}$ de l'apex ; œsophages musculaire

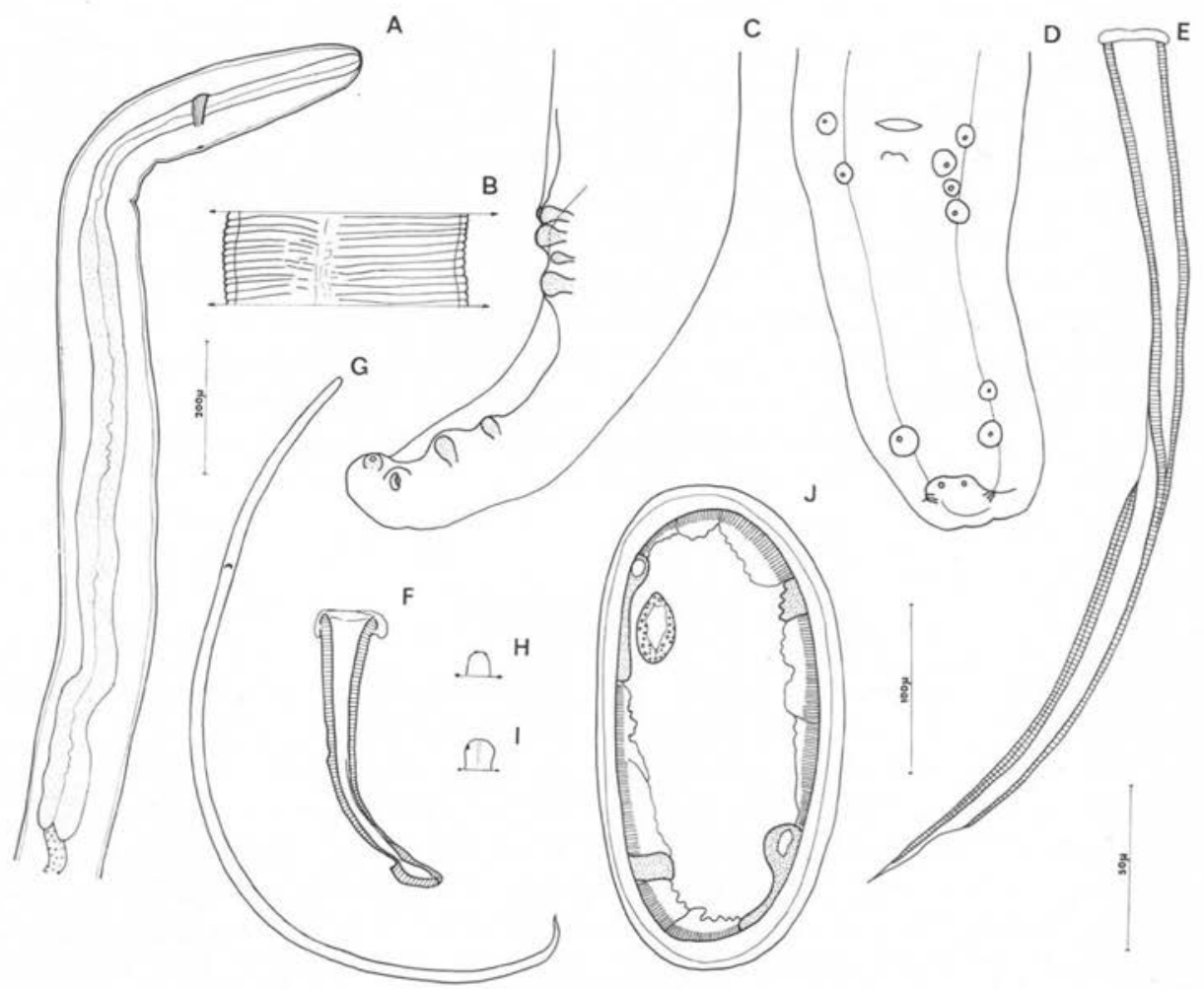

Fig. 5. - Onchocerca lerouxi n. sp. Mâle. A : région antérieure, vue latérale; B, cuticule, au milieu du corps, vue latérale; C et D : queue, vues latérale et ventrale; E : spicule gauche, vue latérale; F : spicule droit, vue latérale; G : microfilaire utérine; H et I : idem, tête, vue opposée au crochet et vue avec crochet latéral; J : femelle, coupe transversale du corps, région moyenne.

(A : éch. $200 \mu ; \mathrm{B}$ et $\mathrm{J}$ : éch. $100 \mu ; \mathrm{C}, \mathrm{D}, \mathrm{E}$ et $\mathrm{F}$, éch. $50 \mu$ ). 
et glandulaire longs de 500 et $960 \mu \mathrm{m}$. La cuticule du corps est ornée de stries profondes, interrompues dans le plan latéral. La queue est longue de $120 \mu \mathrm{m}$; les papilles caudales sont en nombre très réduit et ont une disposition figurée en $5, C$ et $D$.

Le spicule gauche est long de $270 \mu \mathrm{m}$ avec une lame de $140 \mu \mathrm{m}$ terminée par une pointe allongée; le spicule droit est long de $90 \mu \mathrm{m}$ avec un crochet récurrent bien marqué.

\section{Discussion.}

L'élément le plus caractéristique de cette espèce nous paraît être une torsion du corps sur lui-même : l'ensemble du corps subit un certain nombre de boucles et de spires comme chez la plupart des Onchocerques mais ici, en outre, le corps est spiralé sur lui-même si bien que les lignes latérales sont obliques par rapport aux stries et aux côtes de la cuticule.

L'espèce étant proche de la précédente, les caractères différentiels indiqués pour O. sonini vis-à-vis des autres Onchocerques sont les mêmes.

Cette espèce se distingue de la précédente par le corps plus large et la cuticule plus mince, à deux couches chez la femelle, l'œsophage plus long, la grande réduction des papilles caudales et la microfilaire plus longue mais étroite (3 à $3,5 \mu \mathrm{m}$ ), aux pièces céphaliques extrêmement réduites.

Ce matériel constitue donc une nouvelle espèce que nous dédions à la mémoire du $\mathrm{D}^{\mathrm{r}}$ Le Roux, en la nommant $O$. lerouxi n. sp. Cette espèce est caractérisée par la torsion en spirale du corps, autour de l'axe longitudinal, la cuticule à côtes droites avec quatre stries par intercôte, l'œsophage long (1 800-2 $000 \mu \mathrm{m}$ chez la femelle), les papilles caudales en nombre très réduit, la microfilaire fine, longue de 255 à $290 \mu \mathrm{m}$ aux pièces céphaliques extrêmement exiguës.

\section{Onchocerca baeveri n. sp.}

Cette Onchocerque a été trouvée chez deux Cephalophus monticola, l'une tuée à M'Besse (132 PB), l'autre sur la route de Makokou à Okondja (302 PB).

Les adultes sont logés au niveau de l'articulation atlas-occiput ; elles sont situées sur et dans les deux ligaments qui unissent les surfaces articulaires postérieures.

Chez 132 PB, le paquet scléreux s'insinue un peu dans l'axe rachidien mais les méninges autour du bulbe sont d'aspect normal. Chez 302 PB, le kyste est un peu plus gros et plus ou moins calcifié ; la lame fibreuse qui relie postérieurement l'atlas à l'occiput est entièrement prise et la sclérose descend dans le canal rachidien sur presque toute la hauteur de l'atlas. Chez cette même Céphalophe, trois autres kystes plus petits sont récoltés dans la nuque, plus superficiellement; ils sont attenant à des tendons qui lient les muscles postérieurs du cou à l'occiput, long d'environ $1 \mathrm{~cm}$ et larges de $3 \mathrm{~mm}$, jaune rougeâtre et d'aspect un peu grumeleux mais difficiles à voir. 


\section{Matériel :}

Une longue portion antérieure de femelle avec microfilaires (holotype), cinq régions antérieures de femelle, une longue région postérieure et de nombreux fragments de femelle, lot 132 PB; un mâle en deux morceaux (paratype) et des fragments de femelle, lot 302 PB.

Femelle ( $f i g .6)$.

La région antérieure est très grêle $(90 \mu \mathrm{m})$ à la fin de l'œsophage musculaire et peu ondulée sur $1 \mathrm{~cm}$ de long environ. Le corps lui-même est large de $300 \mu \mathrm{m}$ et il décrit de nombreuses boucles.

La cuticule, dans la région moyenne du corps, est épaisse de 12 à $25 \mu \mathrm{m}$; la couche externe forme des côtes peu saillantes, larges de 8-10 $\mu \mathrm{m}$, espacées d'une trentaine de $\mu \mathrm{m}$; la couche interne qui fait la moitié ou les deux tiers de l'épaisseur de la cuticule, forme des stries, au nombre de trois par intercôte (fig. $6 \mathrm{I}$ ).

La musculature est peu épaisse, plus réduite sur la face dorsale; les canaux excréteurs sont volumineux ( $f$ ig. $7 \mathrm{~J}$ ).

Dans la région antérieure, jusqu'à environ $2 \mathrm{~cm}$ de la tête, et dans la région caudale, la cuticule n'est formée que par une couche et les côtes sont très serrées.

Les papilles céphaliques sont disposées suivant un rectangle étiré latéralement, les papilles labiales externes presque suivant un carré (fig. $6, B, C$ et $D$ ). Renflement cervical présent. EEsophage à portion glandulaire bien différenciée. Vulve à la hauteur du passage œsophage musculaire-œsophage glandulaire.

Queue droite, tubulaire, légèrement tordue suivant l'axe longitudinal; l'extrémité caudale porte un sillon transversal qui la sépare en deux lobes, un ventrai et un dorsal plus long; les phasmides se terminent sur le bord ventral du sillon et sont marquées par un mucron saillant ; le bord interne du lobe dorsal est doublé par une saillie cuticulaire médiane (fig. $6 \mathrm{~J}$ à $M$ ).

Dimensions. - Femelle holotype : longueur inconnue; anneau nerveux, pore excréteur et vulve à 180,210 et $440 \mu \mathrm{m}$ de l'apex; œsophages musculaire et glandulaire longs de 465 et $900 \mu \mathrm{m}$.

Autres femelles : anneau nerveux à 180-205 $\mu \mathrm{m}$ de l'apex; vulve à 430-545 $\mu \mathrm{m}$; œsophages musculaire et glandulaire longs de 475-525 $\mu \mathrm{m}$ et 1005-1 $270 \mu \mathrm{m}$. Queue longue de $235 \mu \mathrm{m}$, large de $75 \mu \mathrm{m}$ à l'anus.

Microfilaires utérines ( $f$ ig. $7, K$ à $M$ ).

Elles sont longues de 248 à $262 \mu \mathrm{m}(248,250,253,256,260,262 \mu \mathrm{m})$. Le corps est grêle $(3,5 \mu \mathrm{m})$, à peine plus étroit dans la région céphalique ; la tête porte un sillon transversal subterminal, dont le bord porte un petit crochet ; l'apex porte deux petits renfoncements cuticulaires (quand le crochet est vu de face, un seul est visible). La queue est courte et pointue. 


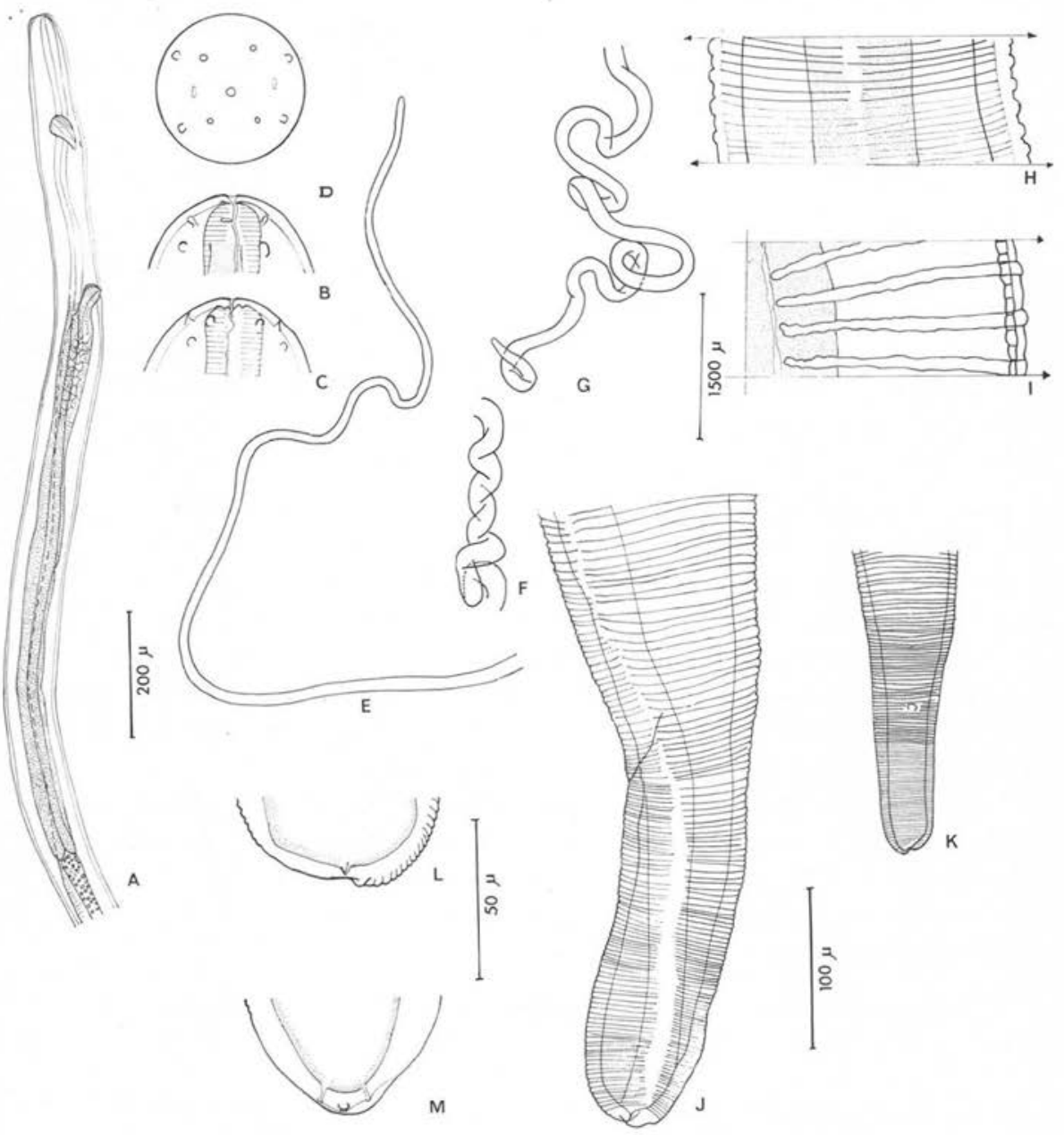

Fig. 6. - Onchocerca beaveri n. sp.; femelle. A : région antérieure, vue latérale; B, C et D, tête, vue latérale, médiane et apicale; E, F, G : silhouettes de la région antérieure, du milieu du corps et de la région postérieure; $\mathrm{H}$ : ornementation cuticulaire, vers le milieu du corps, vue latérale (côtes non représentées de face); I : idem : autre région moyenne du corps, vue latérale (stries non représentées de face); $J$ et $\mathrm{K}$ : queue, vue latérale et ventrale; $\mathbf{L}$ et $\mathbf{M}$ : extrémité caudale, vues latérale et ventrale.

(A et $\mathrm{K}$ : éch. $200 \mu ; \mathrm{B}, \mathrm{C}, \mathrm{D}, \mathrm{L}, \mathrm{M}$ : éch. $50 \mu$; E, F et G : éch. $1500 \mu$; H, I, J : éch. $100 \mu)$. 

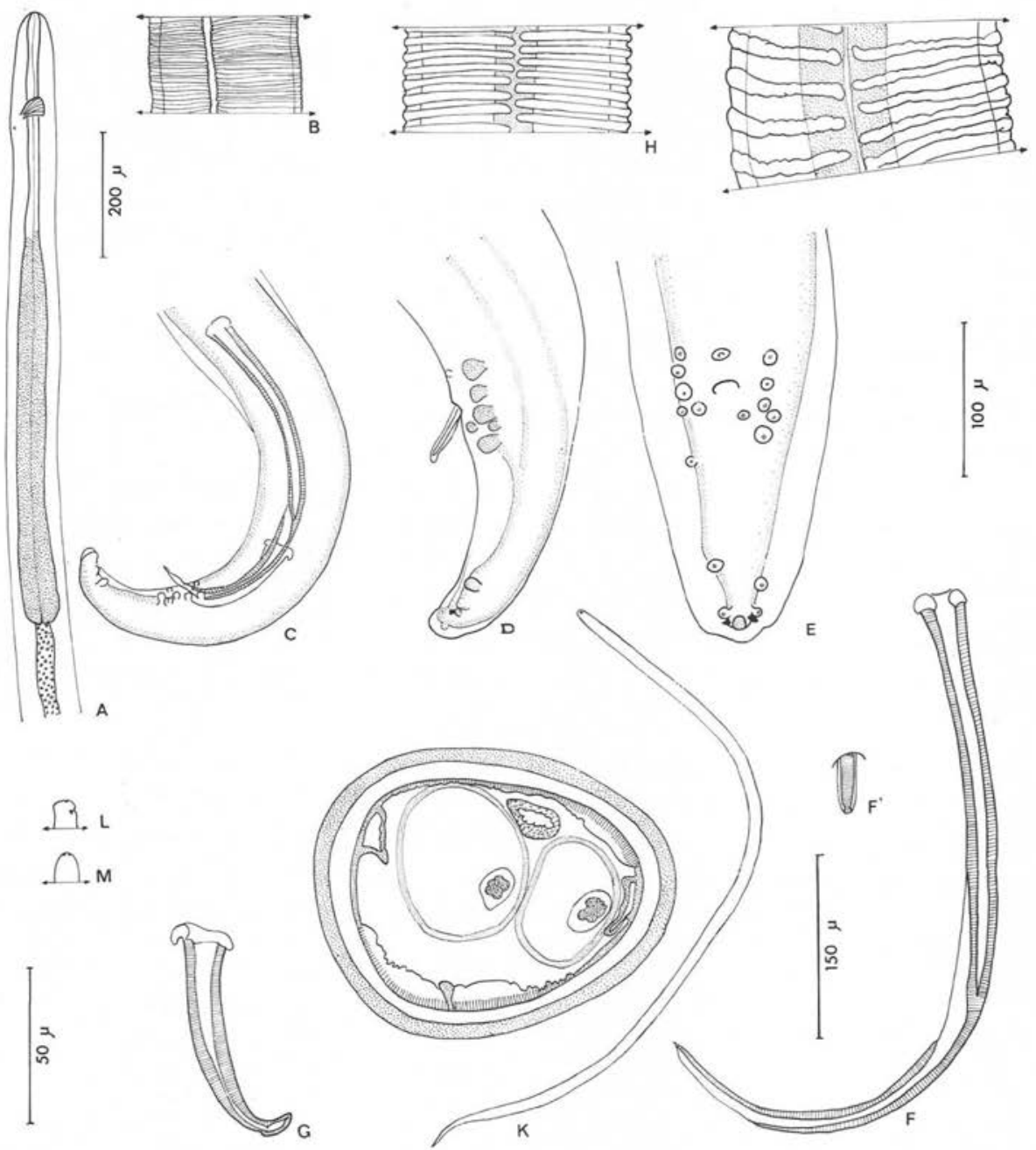

FIG. 7. - Onchocerca beaveri n. sp. A à G, mâle. A : région antérieure, vue latérale ; B : cuticule, milieu du corps, vue latérale; $\mathrm{C}$ : région caudale, vue latérale; $\mathrm{D}$ et $\mathrm{E}$ : queue, vues latérale et ventrale; F : spicule gauche, vue presque latérale; F' : idem, apex, vue ventrale; $\mathrm{G}$ : spicule droit, vue latéro-dorsale; $\mathrm{H}$ à $\mathrm{J}$ : femelle ; $\mathrm{H}$ et $\mathrm{I}$ : cuticule à $1 \mathrm{~cm}$ et $2 \mathrm{~cm}$ de la tête ; $\mathrm{J}$ : coupe transversale du corps; région moyenne; $\mathrm{K}$ : microfilaire utérine; L et M : tête de la microfilaire dans le plan du crochet et perpendiculairement

(A : éch. $200 \mu$; B, C, H, I : éch. $100 \mu$; D, $, \mathrm{E}, \mathrm{F}, \stackrel{\text {, }}{\text {, G }}$ et $\mathrm{K}$ : éch. $50 \mu$; L et M à main levée.) 
MâLe (fig. 7 A à $G$ ).

Corps long de $20,1 \mu \mathrm{m}$ et très fin (98 $\mu \mathrm{m}$ de large). Renflement cervical présent mais moins marqué que chez la femelle. Anneau nerveux et pore excréteur à 150 et $190 \mu \mathrm{m}$ de l'apex ; œsophages musculaire et glandulaire longs de 350 et $630 \mu \mathrm{m}$; queue longue de $80 \mu \mathrm{m}$ avec disposition des papilles figurées en $7, E$; la dernière paire est transformée en pointe cuticulaire; spicule gauche long de $252 \mu \mathrm{m}$ (manche de $145 \mu \mathrm{m}$ ) et à extrémité obtuse ; spicule droit long de $85 \mu \mathrm{m}$ sans crochet marqué. Cuticule du corps ornée de petites côtes très serrées, interrompues dans le plan latéral.

\section{Discussion.}

Ce matériel est proche des deux espèces précédentes mais il s'en distingue aisément :

- il s'oppose à $O$. sonini par la microfilaire beaucoup plus mince et sans capuchon curticulaire, par la cuticule de la femelle plus mince et formée de deux couches seulement ;

- il s'oppose à $O$. lerouxi par l'absence de torsion spiralée longitudinale du corps de la femelle, les papilles caudales plus nombreuses, le mâle plus mince, la microfilaire un peu plus courte et un peu plus épaisse au crochet céphalique plus visible.

Ces spécimens constituent une espèce nouvelle que nous dédions au $\mathrm{P}^{r} \mathrm{P} . \mathrm{C}$. Beaver, en la nommant $O$. beaveri n. sp.

Elle se caractérise par la grande minceur de l'extrémité antérieure de la femelle et du mâle, l'existence de trois stries par intercôte chez la femelle, la dernière paire de papilles caudales transformées en pointes cuticulaires et la microfilaire fine à crochet visible.

\section{Conclusion}

Par leur localisation et leur aspect général, ces trois Onchocerques paraissent a priori très différentes les unes des autres. L'analyse morphologique montre cependant un bon nombre de points communs qui sont : un œsophage bien divisé et moyennement allongé, une cuticule ornée de côtes simples et droites, une vulve plutôt antérieure, des papilles caudales du mâle caractérisées par une forte régression des papilles précloacales s'opposant à la persistance de quatre paires terminales, la dernière étant généralement transformée en une pointe cuticularisée.

Cet ensemble de caractères ne se retrouve pas chez les autres Onchocerques et montre bien que nous avons un groupe homogène. 
Globalement on peut admettre qu'elles sont un peu plus évoluées que la plupart des Onchocerques d'Equidés (œsophage moins long, vulve un peu moins antérieure) mais elles paraissent plus primitives que la plupart des Onchocerques décrites chez les autres Ruminants (œsophage à portion glandulaire bien différenciée, vulve moins postérieure, papilles cloacales terminales bien conservées).

\section{Bibliographie}

Bain O., Muller R.L. (1978): Examen de quelques spécimens d'Onchocerques animales récoltées par le $\mathrm{D}^{r}$ Le Roux. Notes et informations. Ann. Parasitol. hum. comp. (sous presse).

Bain O., Muller R. L., Khamis Y., Guilhon J., Schillhorn Van Veen T. (1976) : Onchocerca raillieti n. sp. (Filarioidea) chez l'Ane domestique en Afrique. J. Helmint., 50, 287-293.

Bain O., Schulz-Key H. (1974): Les Onchocerques du Cerf européen: redescription d'O. flexuosa (Wedl, 1856) et description d'O. tubingensis n. sp. et d'O. tarsicola n. sp. Tropenmed. Parasit., $25,437-449$.

Laurence B. R., Simpson M. G. (1968) : Cephalic and pharyngeal structures in microfilariae revealed by staining. J. Helmint $42,309-330$.

Le Roux P. L. (1947) : Démonstration : 4. Portion of a female of an Onchocerca sp. from a subcutaneous nodule in the region of the brisket in a small African antelope, a duiker (Cephalophus grimmi) at Nteko, Isoka district, N. Rhodesia. Trans. Roy. Soc. Trop. Med. Hyg., 41, 8.

Railliet A., Henry A. (1910) : Les Onchocerques, Nématodes du tissu conjonctif C.R. Soc. Biol., 58, 248-251.

Sonin M. D. (1975): Osnovi Nemaologii, 24, Filariati, Onchocercini. Acad. Sc. Edit., Moscou, $396 \mathrm{p}$. 